\title{
Advantages of "DAW" Composing Music for the Effectiveness of Learning the Process of Musical Practice
}

\author{
Kurnia Eka Fajar*, Yudi Sukmayadi \\ Sekolah Pascasarjana \\ Universitas Pendidikan Indonesia \\ Bandung, Indonesia \\ *sakeeew@gmail.com
}

\begin{abstract}
Digital audio workstation (DAW) is an electronic device or application software used to record, edit, and produce audio files. DAW comes in a variety of configurations from a single software program on a laptop or computer, to an integrated standalone unit, to a highly complex configurations of various components controlled by the central computer. Regardless of configuration, the modern DAW has a central interface that allows users to convert and merge multiple recordings and tracks into the final part produced. In the process of making it, musical works are designed according to what the composer wants based on the structured imagination poured into the DAW to facilitate the composition of the music. One of the advantages is that we get the "shadow sound" that occurs in the work at the evaluation stage before going into the final stage of the composition, but does not close the possibility of revisions to the work due to personal needs. So that we can understand in more detail the composition associated with the learning process.
\end{abstract}

Keywords-composition, DAW, music, effective, practice music

\section{INTRODUCTION}

In an era of significant modernization in terms of its development, there are still some questions we can find about how often questions from composers and artists regarding Digital Audio Workstations (DAW) can organize everything and be made for all purposes. Unfortunately, this is far from reality. Each DAW has different goals, intentions, and functions. There is a huge amount of overlap throughout daw (they all basically do the same), but each nuance is useful when writing for a particular medium and style. DAW is very important in the arrangement of music production in terms of learning as well as professional goals. Of course, we must also be aware of the expanded capabilities of this instrument maker. This allows you to record vocals, compose moving musical compositions and learn learning skills in making music. Mark Marrington [1], Software environments for music production, or Digtal Audio Workstaions (DAW), have since the early 2000s become central to the creation of commercially released music, as indispensable to the studio-based professional recording engineer as to the so-called "bedroom" producer. It proves that music digital production can be done anywhere and anytime in line with the use of time and ideas.

Technological advances in the music industry have unleashed a phenomenon in the application of Digital Audio Workstation (DAW) among them artists in the task of composing, recording, mixing and mastering music. A computer-based DAW is an electronic system consisting of basic components such as audio interfaces, computers, digital audio editor software, input devices, designed to record, edit, and play digital audio. DAW enables recording, editing and mixing audio through a fully digital visual interface, providing the highest sound quality needed to be able to record audio, notation/in the Musical Instrument Digital Interface (MIDI), organizing music data, and exporting in various audio formats.

Each DAW includes built-in plugins for mixing, such as reverb, compressor, and equalizer (EQ), etc. Since the image used to find the edit point is simply a map that tells you that the original data is not permanently altered and the edits can be canceled, something is impossible when cropping the recording. Non-destructive edits are also one of the important improvements enabled by digital audio work stations.

\section{Music Creation Using Digital Audio Workstation (DAW)}

The stages in music making related to technology certainly have their own level of difficulty depending on what we will create and learn in advance the usefulness of the software to be used. The main capital in the process of making music using Digital Audio Workstation (DAW) technology in the current era is intent and tenacity in learning something based on knowledge and musical skills. Only a few people realize how important a composer or artist can compose or create a work in digital form and can express his or her desires in digital form according to imagination and sound shadows based on his experience for the learning needs of practicing music and 
leading to the end result of the work of music that allows for changes/revisions to the work.

Regardless of the selection of a resulting musical material into a digital package, it will again depend on the purpose of a composer creating according to thought and imagination. An example that we can use in the use of Digital Audio Workstation (DAW) software that is quite familiar is the product of Avid namely Sibelius, the feature is in order to process sounds in various instruments in the form of block notation that serves as a shadow of how we write rhythms, melodies, harmonies and other musical elements so that we can listen directly to the processing process at each stage of its creation.

On the other hand, the advantages can be an alternative that is appropriate enough to be applied to the effectiveness of learning in a musical training process where the work can be heard and read directly. Mark Marrington A DAW's essential functionality, when reduced to its simplest terms, is to allow for the manipulation of two main forms of information, MIDI data and digital audio [2].

Some common hardware and software components are obviously very needed given their usefulness in terms of digital use such as computers, loudspeakers, MIDI Controller Keyboards, Microphones, Monitor Headphones, Soundcards (Audio MIDI Interfaces) which are then arranged like figure 1:

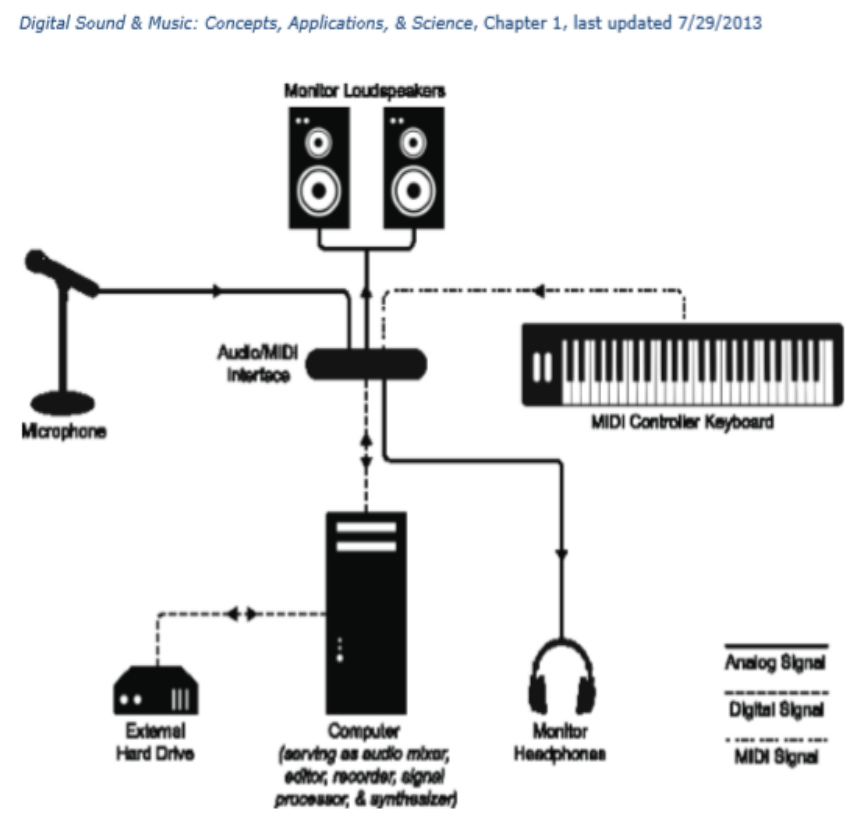

Fig. 1. Basic settings and signal flow of the Digital Audio Workstation component [3].

After the various components that we want to use, there are several software options that can be used in processing digital form music for example: Sibelius, FL Studio, Ableton Live, Cubase, Propellerhead Reason, Cakewalk Sonar, Audacity, Logic Pro X, Reaper, etc. Software selection is tailored to the needs and objectives of composers and artists related to what to do. The built-in tutorial features of the software are very important as additional teaching materials to get to know the system of work in using the software. As an excerpt from the DAW Guidlines for Music Production manual, Charles Dye [4] that:

"There are many similarities between each of the digital audio workstations represented here, and this is what makes these universal guidelines possible. But there are some aspects of each platform where they have their own terminology to describe similar functions or operations."

This quote has a meaning aimed at the user so as not to worry too much about what software to use because almost all of the software has functions or operating systems that are not much different from others.

Here's an example of daw software called Reaper with some elements needed in the music production process in figure 2:

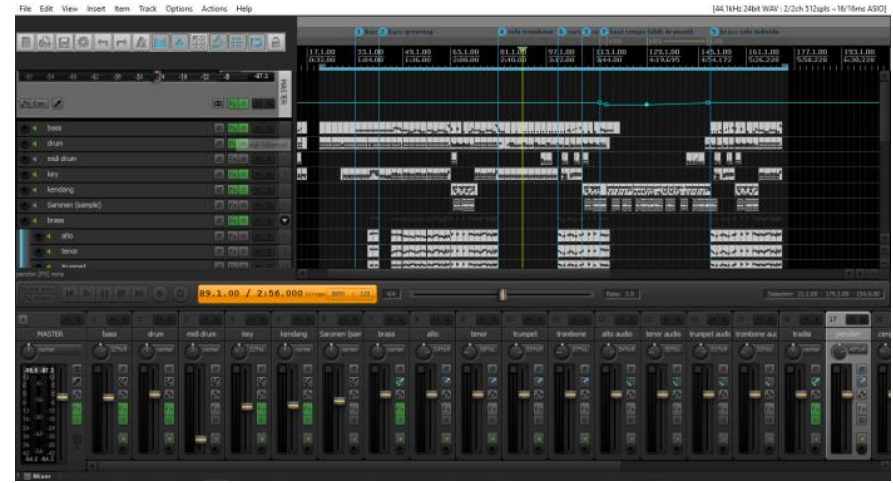

Fig. 2. Reaper basic view (photo: private collection)

In the display there are several elements including: VST Plug-in, MIDI, Mixer, and many supporting features in the process of creating a music project. Joe Bennet, the most common laout of a DAW screen display is in a twodimensionall grid, the horizontal axis representingtime, scrolling from left to right during record or playback, and the vertical axis representing individual sounds-audio or instrument 'tracks' [5].

\section{Music Training Learning Process Using Digital AUDIO WORKSTATION (DAW)}

The learning process that we are used to feeling directly or face-to-face becomes a very visceral culture, but with the technology in this day and age, learning can be improved by using communication media from gadgets or other electronic devices. People's view of technology when it comes to music makers with DAW may cause fear or anxiety where users have to master the field of electronics and all sorts of ways the system works, but on the other hand from a positive point of view, a technology designed to facilitate and have effectiveness in terms of work systems related to its use that we can associate with the learning process of music training. 
The advantages of making a musical work using DAW can be seen from 2 aspects, namely:

\section{A. Audio File Shape}

We can review the design of the music work we are creating with the built-in DAW feature and the end result can be heard directly either in the software or converted into an audio file form, such as; wav, mp3, ddp, flac, mpeg, etc.

\section{B. Display Shapes}

Change the design of music into a physical form by printing with paper or converted into such a file; .sib, .pdf, .wmv (video) include a form of notation that we can review or read for the learning process of music training.

1) Music learning process scheme using Digital Audio Workstation (DAW)

- Starting with the selection of instruments /VST Plug-ins according to the wishes of a composer or artist tailored to the needs of a music project. We can take the built-in instruments available or associated with some additional software because the built-in features are not satisfactory enough because it is lacking in providing various sound samples e.g. kontakt software that has a function as a sound sample storage medium. The instrument column view in figure 3 .

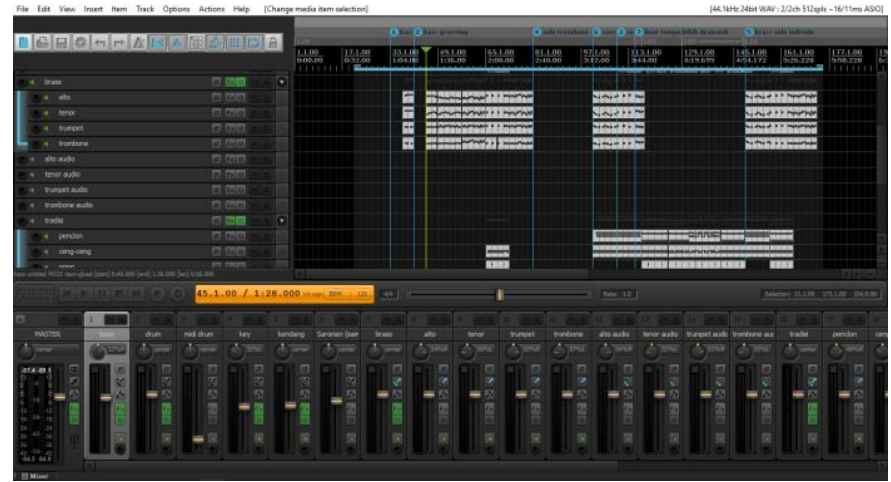

Fig. 3. Instrument column view (photo: private collection).

- Then the tone processer uses MIDI (Musical Instrument Digital Interface). In this section, it is very important to prioritize the difficulty in placing a tone because it will affect the notation so that the player or the reader can understand the melodic sentences composed. There is a velocity feature that has a function to regulate the dynamics of the resulting sound adapted to the interpretation of a composer or artist. The Instrument MIDI display show in figure 4.

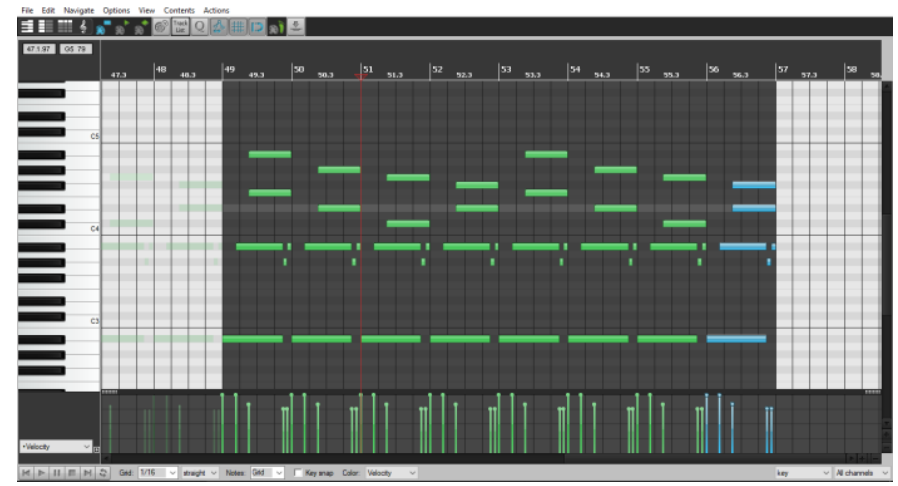

Fig. 4. Instrument MIDI display (photo: private collection).

- In addition to the MIDI display, the processing of tones using DAW can be seen in the form of notation in anticipation of how effective the notation is when reviewed or read by the player at the end later. Musical notations display show in figure 5 .

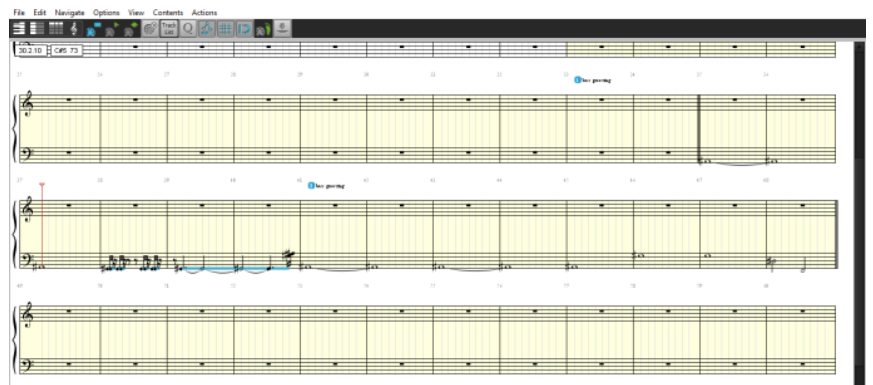

Fig. 5. Musical notations display (photo: private collection)

- For sound capacity setting can use the mixer feature to adjust the size or small sound of the resulting instrument. The Mixer display show in figure 6.

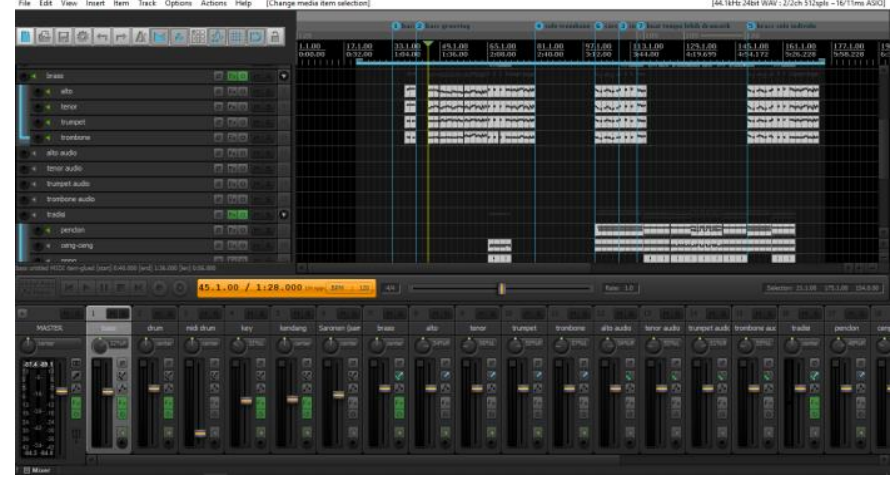

Fig. 6. Mixer display (photo: private collection).

2) Results changed in view form: With this form of physical appearance it is expected that the player or reader can review and analyze directly where this notation is played included audio files that have been prepared to support the sound shadow that occurs in the work related to the 
effectiveness in learning the process of music practice. The Sibelius worksheet show in figure 7.

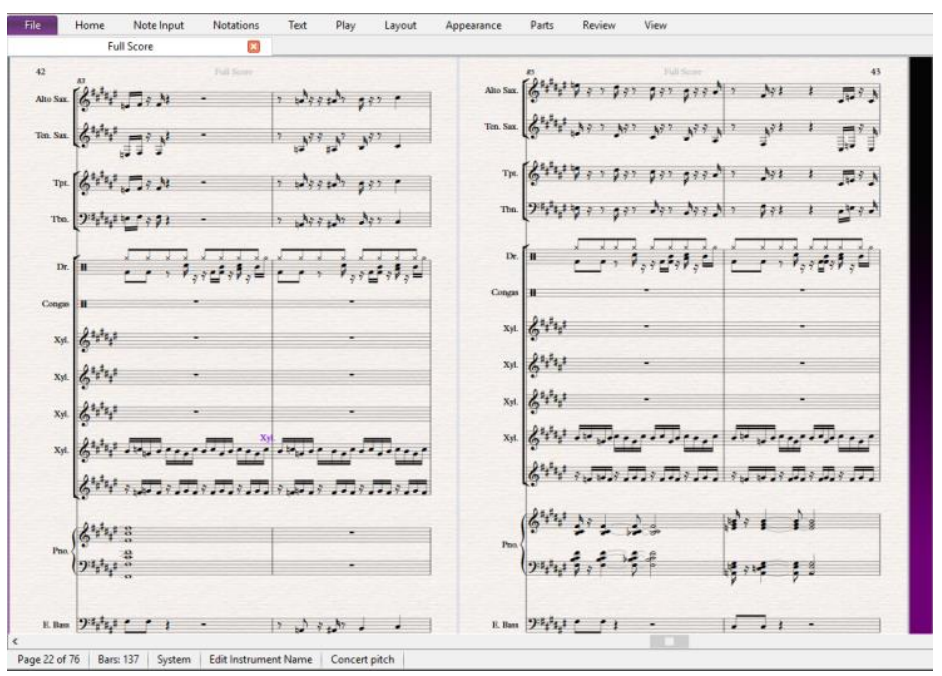

Fig. 7. Sibelius worksheet (photo: private collection).

In addition to being converted into sibelius display form, physical display form files can be converted into pdf files so that they can be printed into physical forms of paper. The notation sheet in pdf file form like a figure bellow.

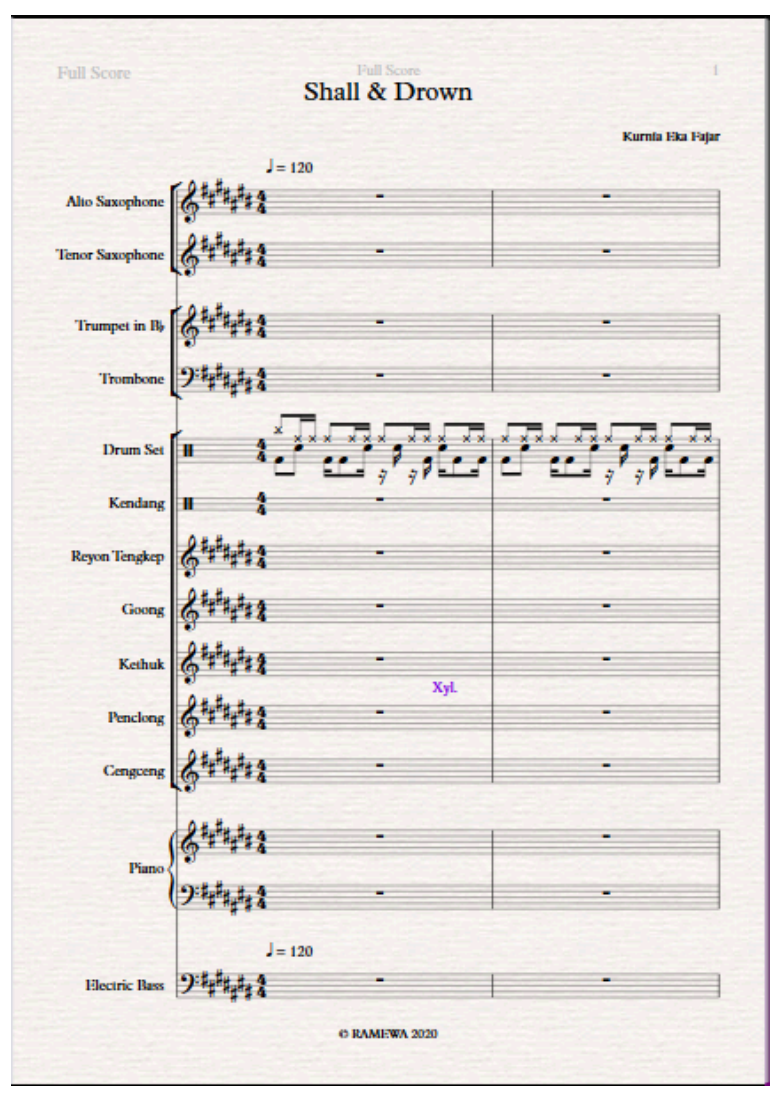

Fig. 8. Notation sheet in pdf file form (photo: private collection)

\section{CONCLUSION}

In principle in learning digital support tools such as Digital Audio Workstation (DAW) is not easy, it takes literacy and guidance from some people who are already in the world of digital music. References to the introduction of software should be honed as technology develops rapidly. Technology in the field of music is used as an effective advantage in terms of time efficiency in the process of learning music practice. The benefits of music technology using Digital Audio Workstation (DAW) can be felt with the help of audio and notation that makes it easier for the player or reader of the work to better understand the work in detail from a composer or artist.

\section{REFERENCES}

[1] M. Marrington, Composing with the Digital Audio Workstation, in J Williams and K. Williams (eds), The Singer Songwriter Handbook. New York: Bloomsbury Academic, 2017, pp. 77-89.

[2] M. Marrington, Paradigms of Music Software Interface Design and Musical Creativity, in R. Hepworth-Sawyer, J. Hogson, J. L. Paterson and R. Toulson eds, Innovation in Music II. Shoreham-by-sea: Futtre Technology Press, 2016, pp 52-63.

[3] J. Burg, J. Romney and E. Schwartz, "Computer science" big ideas" play well in digital sound and music,"In Proceeding of the 44th ACM technical symposium on Computer science education, 2013, pp. 663 668.

[4] C. Dye, DAW Guidlines for Music Production Committee Chairman. Producers \& Engineers Wing of The Recording Academy, 2008.

[5] J Bennet, Songwriting, Digital Audio Workstations, and the Internet Oxford Handbook of Creative Process in Music. Oxford University Press: Oxford, 2018. 